\title{
Coordinated optimization for controlling short circuit current and multi-infeed DC interaction
}

\author{
Dong YANG, Kang ZHAO, Yutian LIU (ه)
}

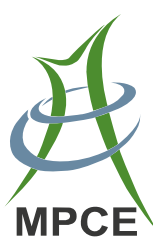

\begin{abstract}
Due to increased penetration of renewable energies, DC links and other emerging technologies, power system operation and planning have to cope with various uncertainties and risks. In order to solve the problems of exceeding short circuit current and multi-infeed DC interaction, a coordinated optimization method is presented in this paper. Firstly, a branch selection strategy is proposed by analyzing the sensitivity relationship between current limiting measures and the impedance matrix. Secondly, the impact of network structure changes on the multi-infeed DC system is derived. Then the coordinated optimization model is established, which considers the cost and effect of current limiting measures, the tightness of network structure and the voltage support capability of AC system to multiple DCs. Finally, the non-dominated sorting genetic algorithm II combining with the branch selection strategy, is used to find the Pareto optimal schemes. Case studies on a planning power system demonstrated the feasibility and speediness of this method.
\end{abstract}

Keywords Operation and planning, Multiple DC infeed, Short circuit current, Sensitivity analysis, Multi-objective optimization

CrossCheck date: 13 November 2014

Received: 10 September 2014/ Accepted: 13 November 2014/ Published online: 5 December 2014

(C) The Author(s) 2014. This article is published with open access at Springerlink.com

D. YANG, State Grid Shandong Electric Power Research Institute, Jinan 250002, China

K. ZHAO, Y. LIU, Key Laboratory of Power System Intelligent

Dispatch and Control of Ministry of Education,

Shandong University, Jinan 250061, China

$(\bowtie)$ e-mail: liuyt@sdu.edu.cn

\section{Introduction}

Modern power system operation and planning is undergoing dramatic changes. Due to increased penetration of renewable energies, DC systems and other emerging technologies, system operation and planning must now cope with various uncertainties and risks. Most of them belong to the multi-objective, non-linear, non-convex and mixedinteger programming problem. It calls for effective solutions to coordinate and optimize new and old technologies to improve overall system security and efficiency at large. The traditional optimization techniques are usually inefficient or even unable to handle these problems. Fortunately, the rapid development of modern optimization techniques provides the promising way to solve the difficulties and challenges in modern power system environments [1-5].

Exceeding short circuit current and multi-infeed DC interaction are two problems faced by the receiving-end power grid in China. Coordinated optimization for them is a typical multi-objective problem. The classical optimization algorithms suggest converting the multi-objective problem to a single-objective problem. When such an algorithm is applied to find multiple solutions, it has to be carried out many times, hopefully finding a set of optimal solutions. This process is usually inefficient and cost a lot of time. Recently, the emergence of evolutionary algorithms (EAs) can effectively solve this problem [6,7]. EAs are well applicable to multi-objective problems because of their ability to find multiple solutions in one single simulation run. In the family of EAs, the nondominated sorting genetic algorithm II (NSGA-II) outperforms others in terms of finding a diverse set of solutions and converging near the true Pareto optimal solutions [7].

Short circuit current can cause mechanical and thermal stresses proportional to the square of the current, and hence lead to the damage of the equipment in power system. This situation is worsened with the increase of interconnections 
in power system and high-capacity generators being injected to the grid. Short circuit current can be limited in many ways, which can be divided into two categories depending on the cost and effect. One is to open the switches of lines or buses, which is simple, economical and obviously effective. However, this method has a greater impact on the system stability. The other is to increase the installations of electrical equipment or upgrade them, such as installing the current limiting reactor or fault current limiter (FCL), even replacing with the high-impedance transformer. This method has little influence on the system stability, but the high cost is needed and the effect is limited.

Comparing various current limiting measures one by one is the traditional way to solve the problem of exceeding short circuit current, which is tedious and inefficient. When the requirement cannot be satisfied by using one single measure, several measures are integrated based on the engineering experience. The application of current limiting measures is mature, but the optimization of them using mathematical methods is unusual. In [8-11], the optimization for limiting short circuit current was mainly focused on the allocation of FCL installation location, quantity and impedance values. Reference [12] presented a method with genetic algorithm and particle swarm optimization to find the optimal location and the number of buses to be split. In [13], the optimization problem was modeled as a $0-1$ mixed integer programming problem, and the model can be solved by a branch and bound algorithm to generate optimal current limiting strategies.

Multiple DC infeed is another important feature of the receiving-end power grid in China. From theoretical analysis and simulation, it is found that the biggest risk faced by the multi-infeed DC system is the voltage stability problem [14-16]. The short-circuit ratio was given to evaluate the voltage support capability of AC system to DC system in [17]. This index has not considered the interaction of multiple DCs, thus it is applicable to the system with just one DC. Reference [18] suggested an extension of the classical short-circuit ratio to multi-infeed DC systems, named the multi-infeed short-circuit ratio. This new index considers the AC system short circuit capacity, the multiple DC transmission capacity, and the electrical coupling relationship between DC inverter stations. Reference [19] showed that it is effective to use such new index for denoting the voltage stability level of the multi-infeed DC system. Reference [20] predicted the risk for voltage and power instability when the multi-infeed short-circuit ratio is low.

Controlling short circuit current and increasing power system stability is a contradiction [21]. The application of current limiting measures will change the power grid structure, and then affect the voltage support capability of AC system to multiple DCs [22]. Specifically, the current limiting measures, on the one hand, reduce short circuit current, on the other hand, stretch the electrical distance between DC inverter stations. As a result, the multi-infeed short-circuit ratio may be increased or decreased under different conditions. This fact indicates that there exists a coordinated optimization scheme, which can not only control short circuit current within a reasonable range, but also remain the multi-infeed short-circuit ratio at a high level. Such scheme should be obtained by establishing and solving the multiobjective optimization model. Unfortunately, most researches pay attention to the single-objective optimization for the cost of current limiting measures, and there are still no literatures that consider the influence of current limiting measures on the multi-infeed DC system.

A coordinated optimization method for controlling short circuit current and multi-infeed DC interaction is presented in this paper. Firstly, by analyzing the sensitivity relationship between current limiting measures and the impedance matrix, a branch selection strategy is proposed. Secondly, the impact of network structure changes on the multi-infeed DC system is derived. Then the coordinated optimization model, which considers the cost and effect of current limiting measures, the tightness of network structure and the voltage support capability of AC system to multiple DCs, is established. Finally, combining NSGA-II with the branch selection strategy, the Pareto optimal schemes are found. Taking the regional power grid in China for example, the feasibility and efficiency of the method is validated.

\section{Current limiting measures sensitivity analysis}

Opening the line and installing the current limiting reactor are two typical current limiting measures. Therefore, they are used in this paper to solve the optimization problem.

Three-phase short circuit is generally the most serious short circuit fault in power systems, and usually used to determine the rated breaking capacity of circuit breakers. Three-phase short circuit current is inversely proportional to the self-impedance. The following is the derivation of sensitivity relationship between current limiting measures and the self-impedance of overproof site. Assuming the original network forms an $m$-order impedance matrix $\boldsymbol{Z}_{m}$. If the branch $z_{i j}$ is added into the network between node $i$ and $j$, the impedance matrix changes into $\boldsymbol{Z}_{m}^{\prime}$. According to the additional branch method, the element in matrix $\boldsymbol{Z}_{m}^{\prime}$ can be derived as

$Z_{k l}^{\prime}=Z_{k l}-\frac{\left(Z_{k i}-Z_{k j}\right)\left(Z_{l i}-Z_{l j}\right)}{Z_{i i}+Z_{j j}-2 Z_{i j}+z_{i j}}$

where $k=1,2, \ldots, m ; l=1,2, \ldots, m ; m$ is the number of network nodes; $Z_{k l}^{\prime}$ and $Z_{k l}$ are the element of the impedance matrix $\boldsymbol{Z}_{m}^{\prime}$ and $\boldsymbol{Z}_{m}$, respectively. 


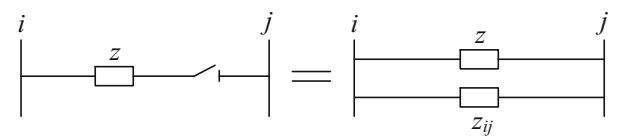

(a)

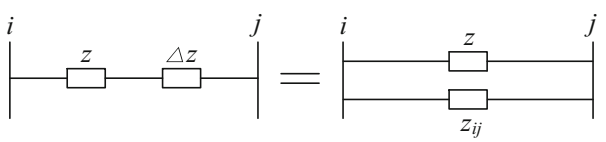

(a) (b)
Fig. 1 Equivalent model of opening a line

\subsection{Open the line}

Opening a line is equivalent to adding a branch $z_{i j}=-z$, between node $i$ and $j$, which is shown in Fig. 1 .

If two buses connected by a bus coupler switch are regarded as independent nodes, splitting the buses is similar to opening a line. The sensitivity of opening the line with respect to the self-impedance is defined as

$$
\left\{\begin{array}{l}
\lambda_{k}=\frac{Z_{k k}^{\prime}-Z_{k k}}{Z_{k k}}=\frac{\Delta Z_{k k}^{(1)}}{Z_{k k}} \\
\Delta Z_{k k}^{(1)}=-\frac{\left(Z_{k i}-Z_{k j}\right)^{2}}{Z_{i i}+Z_{j j}-2 Z_{i j}-z}
\end{array}\right.
$$

where $Z_{k k}$ and $Z_{k k}^{\prime}$ are the self-impedance of overproof site $k$ before and after opening a line, respectively.

A greater $\lambda_{k}$ indicates a better current limiting effect on overproof site $k$ when opening the line. Considering the current limiting effect on all overproof sites, the weighted sensitivity is defined as

$$
\left\{\begin{array}{l}
\lambda=\sum_{k=1}^{N_{e}} \omega_{k} \lambda_{k} \\
\omega_{k}=\left(\frac{I_{k}}{I_{k}^{\max }}\right)^{2}
\end{array}\right.
$$

where $N_{e}$ is the number of overproof sites; $\omega_{k}$ is the weighted coefficient; $I_{k}$ and $I_{k}^{\max }$ are the actual three-phase short circuit current and the maximum breaker interruptive current of overproof site $k$, respectively.

\subsection{Install the current limiting reactor}

Installing the current limiting reactor is equivalent to adding a branch $z_{i j}=-\left(z^{2}+z \Delta z\right) \Delta z$, between node $i$ and $j$, which is shown in Fig. 2.

The ideal fault current limiter does not affect the normal operation of power systems, and can quickly put into a large current limiting reactor when short circuit fault occurs. Therefore, installing the fault current limiter is similar to installing the current limiting reactor. The sensitivity of installing the current limiting reactor with respect to the self-impedance is defined as
Fig. 2 Equivalent model of installing the current limiting reactor

$$
\left\{\begin{array}{l}
\gamma_{k}=\lim _{\Delta z \rightarrow 0} \frac{\Delta Z_{k k}^{(2)}}{\Delta z}=\left(\frac{Z_{k i}-Z_{k j}}{z}\right)^{2} \\
\Delta Z_{k k}^{(2)}=-\frac{\left(Z_{k i}-Z_{k j}\right)^{2}}{Z_{i i}+Z_{j j}-2 Z_{i j}-\frac{z^{2}+z \Delta z}{\Delta z}}
\end{array}\right.
$$

A greater $\gamma_{k}$ indicates a better current limiting effect on overproof site $k$ when installing the current limiting reactor. Considering the current limiting effect on all overproof sites, the weighted sensitivity is defined as

$$
\left\{\begin{array}{l}
\gamma=\sum_{k=1}^{N_{e}} \omega_{k} \gamma_{k} \\
\omega_{k}=\left(\frac{I_{k}}{I_{k}^{\max }}\right)^{2}
\end{array}\right.
$$

From (2) and (4), it can be concluded that for the same branch, $\Delta Z_{k k}^{(1)}=\Delta Z_{k k}^{(2)}$ when $\Delta z \rightarrow \mathrm{j} \cdot+\infty$ and $\Delta Z_{k k}^{(1)}>\Delta Z_{k k}^{(2)}$ when $\Delta z>\mathrm{j} \cdot 0$. It means that opening the line has a better current limiting effect than installing the current limiting reactor.

\subsection{Branch selection strategy}

As described above, for the same branch, the sensitivity of opening the line reflects the current limiting characteristic with $\Delta z \rightarrow \mathrm{j} \cdot+\infty$; the sensitivity of installing the current limiting reactor reflects the current limiting characteristic with $\Delta z \rightarrow \mathrm{j} \cdot 0$. Thus, the integrated sensitivity of one branch is defined as the average of these two sensitivities.

$\left\{\begin{array}{l}\mu_{l}=\frac{\lambda_{l}^{*}+\gamma_{l}^{*}}{2} \\ \lambda_{l}^{*}=\frac{\lambda_{l}-\lambda^{\min }}{\lambda^{\mathrm{max}}-\lambda^{\mathrm{min}}} \\ \gamma_{l}^{*}=\frac{\gamma_{l}-\gamma^{\mathrm{min}}}{\gamma^{\mathrm{max}}-\gamma^{\mathrm{min}}}\end{array}\right.$

where $\mu_{l}$ is the integrated sensitivity of the branch $l ; \lambda_{l}^{*}$ and $\lambda_{l}$ are the normalized value and actual value of the sensitivity of opening the line for the branch $l$, respectively; $\gamma_{l}^{*}$ and $\gamma_{l}$ are the normalized value and actual value of the sensitivity of installing the current limiting reactor for the branch $l$, respectively.

Sort all the branches of the network in descending order of the integrated sensitivity, choose the first $M$ branches, and then form the reduced-dimensional set of decision variables. 


\section{Multi-infeed short-circuit ratio analysis}

\subsection{Definition of the multi-infeed short-circuit ratio}

The CIGRE DC working group proposed the definition of the multi-infeed short-circuit ratio [18]:

$$
\begin{aligned}
\operatorname{MISCR}_{i} & =\frac{S_{\mathrm{ac} i}}{P_{\mathrm{deq} i}}=\frac{S_{\mathrm{ac} i}}{P_{\mathrm{d} i}+\sum_{j=1, j \neq i}^{n} M I I F_{j i} P_{\mathrm{d} j}} \\
& =\frac{S_{\mathrm{ac} i}}{P_{\mathrm{d} i}+\sum_{j=1, j \neq i}^{n} \frac{\Delta U_{j}}{\Delta U_{i}} P_{\mathrm{d} j}}
\end{aligned}
$$

where $S_{\text {aci }}$ is the inverter bus short circuit capacity of the $i^{\text {th }}$ DC; $P_{\text {deq } i}$ is the equivalent DC transmission capacity; $n$ is the number of DCs; $P_{\mathrm{d} i}$ is the rated transmission capacity of the $i^{\text {th }} \mathrm{DC}$; and $M I I F_{j i}$ is the multi-infeed interaction factor, which is defined as the ratio of the $j^{\text {th }}$ DC inverter bus voltage variation $\Delta U_{j}$ to the $i^{\text {th }}$ DC $\Delta U_{i}$ when a reactive power disturb is applied on the $i^{\text {th }} \mathrm{DC}$ inverter bus.

Another practical definition of the multi-infeed short circuit ratio is based on the impedance matrix [19]. It can be expressed as

$$
M I S C R_{i}=\frac{U_{\mathrm{aci}}^{2} /\left|Z_{\mathrm{eq} i i}\right|}{P_{\mathrm{d} i}+\sum_{j=1, j \neq i}^{n}\left|Z_{\mathrm{eq} i j} / Z_{\mathrm{eq} i i}\right| P_{\mathrm{d} j}}
$$

where $U_{\mathrm{ac} i}$ is the inverter bus voltage of the $i^{\text {th }} \mathrm{DC} ; Z_{\mathrm{eq} i j}$ is the $i^{\text {th }}$ row and $j^{\text {th }}$ column element of the equivalent impedance matrix $\boldsymbol{Z}_{\mathrm{eq}}$, which can be obtained by multi-port Thevenin equivalent method between DC inverter buses. And the value of $Z_{\text {eq } i j}$ is equal to the voltage of node $i$ when the unit current being injected only to node $j$.

If the inverter bus rated voltage is set as the voltage base value, (8) is rewritten as

$$
\operatorname{MISCR}_{i}=\frac{1}{\left|Z_{\mathrm{eq} i i}\right| P_{\mathrm{d} i}+\sum_{j=1, j \neq i}^{n}\left|Z_{\mathrm{eq} i j}\right| P_{\mathrm{d} j}}=\frac{1}{\sum_{j=1}^{n}\left|Z_{\mathrm{eq} i j}\right| P_{\mathrm{d} j}}
$$

3.2 Impact of network structure changes on the multiinfeed short-circuit ratio

Assuming the original network contains $m$ nodes and $n$ DCs, and the first $n$ rows and $n$ columns of the $m$-order impedance matrix $\boldsymbol{Z}_{m}$ are DC inverter buses. When a branch $z_{k l}$ is added between node $k$ and $l$, according to (1) and (9), the multi-infeed short-circuit ratio is changed into

$$
\operatorname{MISCR}_{i}^{\prime}=\frac{1}{\sum_{j=1}^{n}\left|Z_{i j}^{\prime}\right| P_{\mathrm{d} j}}=\frac{1}{\sum_{j=1}^{n}\left|Z_{i j}-\frac{\left(Z_{i k}-Z_{i l}\right)\left(Z_{j k}-Z_{j l}\right)}{Z_{k k}+Z_{l l}-2 Z_{k l}+Z_{k l} \mid}\right| P_{\mathrm{d} j}}
$$

where $i=1,2, \ldots, n ; j=1,2, \ldots, n ; n$ is the number of DCs; $k=1,2, \ldots, m ; l=1,2, \ldots, m ; m$ is the number of network nodes; $Z_{i j}$ and $Z_{i j}^{\prime}$ are the $i^{\text {th }}$ row and $j^{\text {th }}$ column element of the impedance matrix before and after adding the branch, respectively.

The variation of the multi-infeed short-circuit ratio is:

$$
\begin{aligned}
& \Delta M_{I S C R_{i}}=M_{I S C R_{i}^{\prime}}-M_{I S C R_{i}} \\
& =\frac{1}{\sum_{j=1}^{n} \mid Z_{i j}-\frac{\left(Z_{i k}-Z_{i l}\right)\left(Z_{j k}-Z_{j l}\right)}{Z_{k k}+Z_{l l}-2 Z_{k l}+Z_{k l} \mid}-P_{\mathrm{d} j}}-\frac{1}{\sum_{j=1}^{n}\left|Z_{i j}\right| P_{\mathrm{d} j}} \\
& =\frac{\sum_{j=1}^{n}\left(\left|Z_{i j}\right|-\left|Z_{i j}-\frac{\left(Z_{i k}-Z_{i l}\right)\left(Z_{j k}-Z_{j l} \mid\right.}{Z_{k k}+Z_{l l}-2 Z_{k l}+Z_{k l}}\right|\right) P_{\mathrm{d} j}}{\sum_{j=1}^{n}\left|Z_{i j}-\frac{\left(Z_{i k}-Z_{i l}\right)\left(Z_{j k}-Z_{j l}\right)}{Z_{k k}+Z_{l l}-2 Z_{k l}+z_{k l} \mid}\right| P_{\mathrm{d} j} \cdot \sum_{j=1}^{n}\left|Z_{i j}\right| P_{\mathrm{d} j}}
\end{aligned}
$$

The denominator of (11) is greater than 0 . And if the elements of the impedance matrix are regarded as pure inductive reactance, the numerator of (11) can be rewritten as

$$
\left\{\begin{array}{l}
\Delta=\sum_{j=1}^{n} \frac{\left(Z_{i k}-Z_{i l}\right)\left(Z_{j k}-Z_{j l}\right)}{Z_{k k}+Z_{l l}-2 Z_{k l}+z_{k l}} P_{\mathrm{d} j}=\frac{\Delta^{\prime}}{Z_{L L}} \\
\Delta^{\prime}=\sum_{j=1}^{n}\left(Z_{i k}-Z_{i l}\right)\left(Z_{j k}-Z_{j l}\right) P_{\mathrm{d} j}
\end{array}\right.
$$

For opening the line as is shown in Fig. 1, since that line reactance $z$ is usually much larger than the self-impedance, the expression can be derived as

$Z_{L L}=Z_{k k}+Z_{l l}-2 Z_{k l}-z<\mathrm{j} \cdot 0$

For installing the current limiting reactor as is shown in Fig. 2, similarly, the expression can be derived as

$Z_{L L}=Z_{k k}+Z_{l l}-2 Z_{k l}-\frac{z^{2}}{\Delta z}-z<\mathrm{j} \cdot 0$

The $\Delta^{\prime}$ expression shows that its value is determined by the network structure and component parameters. If $\Delta^{\prime}$ is larger than 0 , then $\triangle M I S C R_{i}$ is larger than 0 ; if $\Delta^{\prime}$ is smaller than 0 , then $\triangle M I S C R_{i}$ is smaller than 0 . The current limiting measures, on the one hand, reduce short circuit current, on the other hand, stretch the electrical distance between DC inverter stations. As a result, the multi-infeed short-circuit ratio may be increased or decreased under different conditions. This fact indicates that there exists a coordinated optimization scheme, which can not only control short circuit current within a reasonable range, but also remain the multi-infeed short-circuit ratio at a high level. Such scheme should be obtained by establishing and solving the multi-objective optimization model. 


\section{Coordinated optimization method}

\subsection{Mathematical model}

The decision variables consist of two parts. One is the control variable $u_{s}$ which represents whether the measure is put into or not, and the other is the variable $z_{s}$ which represents the specific parameters of current limiting equipment. The coordinated optimization not only considers the cost and effect of current limiting measures, but also tries to keep the tightness of network structure. Furthermore, the impact of current limiting measures on the multi-infeed short-circuit ratio should be considered.

The objective function $f_{1}$ is used to evaluate the economy of current limiting measures, which is expressed as the total cost

$\min f_{1}=\sum_{s=1}^{N_{s}} u_{s}\left(k_{a s}+k_{b s} z_{s}\right)$

where $N_{s}$ is the number of put-into current limiting measures; $u_{s}=1$ represents that the measure $s$ is put into and $u_{s}=0$ represents that the measure $s$ is not put into; $k_{a s}$ and $k_{b s}$ are the cost factors of the measure $s[13] ; z_{s}$ is the reactance value of the current limiting reactor or the short circuit voltage percentage increment of the high-impedance transformer.

The objective function $f_{2}$ is used to evaluate the tightness of network structure, which is expressed as the short circuit capacity margin:

$\min f_{2}=\sum_{k=1}^{N_{b}} \frac{s c c_{k}^{\max }-s c c_{k}}{s c c_{k}}$

where $N_{b}$ is the total number of nodes; $s c c_{k}^{\max }$ is the short circuit capacity upper limit of node $k$, and less than the maximum breaker interruptive capacity; $s c c_{k}$ is the short circuit capacity of node $k$ after current limiting measures are put into.

The short circuit capacity reflects the anti-disturbance performance of each node and the network connection strength [21]. In this paper, current limiting measures are tried not to destroy the integrity and tightness of power grid. Thus, the minimum short circuit capacity margin is chosen as one target. At the same time, taking the current limiting effect into account, the short circuit current upper limit can be specified according to the engineering experience.

The objective function $f_{3}$ is used to evaluate the impact of current limiting measures on the multi-infeed short-circuit ratio, which reflects the voltage support capacity of AC system to multiple DCs. A greater value of $f_{3}$ indicates a stronger inherent strength of the AC system. It is expressed as the weighted multi-infeed short-circuit ratio: $\max f_{3}=\sum_{i=1}^{n} \omega_{i} M I S C R_{i}$

where $n$ is the number of DCs; $M I S C R_{i}$ is the multi-infeed short-circuit ratio of the $i^{\text {th }} \mathrm{DC} ; \omega_{i}$ is the weighted factor of the $i^{\text {th }} \mathrm{DC}$, which reflects the importance of the $i^{\text {th }} \mathrm{DC}$ in multi-infeed DC systems. The greater influence of the $i^{\text {th }}$ DC on other DCs indicates the more importance of the $i^{\text {th }}$ DC. Thus, $\omega_{i}$ can be defined as:

$\omega_{i}=\sum_{j=1, j \neq i}^{n}\left|\frac{Z_{\mathrm{eq} i j}}{Z_{\mathrm{eq} i i}}\right| \cdot \frac{P_{\mathrm{d} j}}{P_{\mathrm{d} i}}$

The constraints include that there are no isolated node in power grid, active power flow balance, reactive power flow balance, short circuit current within limit, branch power flow within limit, bus voltage within limit, the multi-infeed shortcircuit ratio within limit, parameters of current limiting equipment within limit, etc. They are shown as (19):

$$
\left\{\begin{array}{l}
P_{i}=U_{i} \sum_{j=1}^{N_{b}} U_{j}\left(G_{i j} \cos \delta_{i j}+B_{i j} \sin \delta_{i j}\right) \quad i=1,2, \ldots, N_{b} \\
Q_{i}=U_{i} \sum_{j=1}^{N_{b}} U_{j}\left(G_{i j} \sin \delta_{i j}-B_{i j} \cos \delta_{i j}\right) \quad i=1,2, \ldots, N_{b} \\
I_{k} \leq I_{k}^{\max } \quad k=1,2, \ldots, N_{b} \\
S_{l} \leq S_{l}^{\max } \quad l=1,2, \ldots, N_{l} \\
U_{k}^{\min } \leq U_{k} \leq U_{k}^{\max } \quad k=1,2, \ldots, N_{b} \\
M I S C R_{i} \geq M I S C R_{\min } \\
z_{s}^{\min } \leq z_{s} \leq z_{s}^{\max }
\end{array}\right.
$$

where $N_{b}$ is the total number of nodes; $I_{k}^{\max }$ is the short circuit current upper limit of node $k ; N_{l}$ is the total number of branches; $S_{l}^{\max }$ is the power upper limit of branch $l ; U_{k}^{\max }$ and $U_{k}^{\min }$ are the voltage upper and lower limits of node $k$; $M I S C R_{\min }$ is the lower limit of the multi-infeed short-circuit ratio; $z_{s}^{\max }$ and $z_{s}^{\min }$ are the upper and lower limits of parameters of current limiting equipment.

Additionally, the optimization schemes should meet the "N-1" constraints. In order to simplify the problem, the handing method in this paper is getting the preferred schemes firstly, and then checks them.

\subsection{Multi-objective optimization algorithm}

The core of multi-objective optimization is to coordinate the relationships between objective functions, and to find out the optimal solutions which make the value of each objective function as small as possible. The multi-objective optimization algorithm has three main performance indices: 1) the obtained solutions should be as close to the true Pareto optimal solutions as possible; 2) try to keep the distribution and diversity of the individuals; 3) avoid 
missing the Pareto optimal solutions in the solving process.

The classical optimization algorithms suggest converting the multi-objective problem to a single-objective problem by sorting or assigning weighted factors to multiple objectives. When such an algorithm is applied, only one single optimal solution can be obtained in each simulation run. It has to be carried out many times to find a set of optimal solutions. This process is usually inefficient and cost a lot of time. In addition, the solutions are badly influenced by weighted factors, which are determined according to the expert experience. Thus, these classical algorithms are not quit applicable to multi-objective problems.

Over the past decade, a number of multi-objective EAs have been suggested. EAs are well applicable to multiobjective problems because of their ability to find multiple solutions in one single simulation run. Since EAs work with a population of individuals, it can be extended to maintain a diverse set of solutions. With an emphasis for moving toward the true Pareto optimal region, an EA can be used to find the Pareto optimal solutions in one single simulation run. NSAG-II is one of such EAs and it outperforms others in terms of finding a diverse set of solutions and converging near the true Pareto optimal solutions. NSGA-II has three key technologies, which make it an excellent multi-objective optimization algorithm. They are the fast non-dominated sorting approach, individual crowding distance design and elitist strategy [7].

\subsubsection{Individual encoding}

There may be three states that exist on the same branch, including no current limiting measures applied, opening the line and installing the current limiting reactor. Perform integer encoding on the $N$ individuals of the population. Each individual consists of $M$ bits, which is shown in Fig. 3.

$z_{l}$ is the value of the $l^{\text {th }}$ bit which can be any integer in $\left(0,\left[z_{l}^{\min }, z_{l}^{\max }\right], z_{l}^{\max }+1\right) . z_{l}=0$ denotes no current limiting measure is applied; $z_{l}=z_{l}^{\max }+1$ denotes opening the line for the branch $l$; if $z_{l}$ is any integer in $\left[z_{l}^{\min }, z_{l}^{\max }\right]$, it means installing a current limiting reactor with the value of $z_{l}$ for the branch $l$.

\subsubsection{Fitness function}

Adding the constraints in (19) into the objective functions as a penalty, then the fitness functions can be expressed as

\begin{tabular}{|l|l|l|l|l|l|}
\hline$z_{1}$ & $z_{2}$ & $\cdots$ & $z_{l}$ & $\cdots$ & $z_{M}$ \\
\hline
\end{tabular}

Fig. 3 Integer encoding structure
$\left\{\begin{array}{l}f_{1}=f_{1}+W \\ f_{2}=f_{2}+W \\ f_{3}=-f_{3}+W\end{array}\right.$

If all the constraints are satisfied, the penalty $W$ is assigned to 0 ; otherwise, $W$ is assigned to a large value.

\subsubsection{Fast non-dominated sorting approach}

This approach sorts the individuals into different nondominated fronts, and it guides the search process toward the Pareto optimal solutions. For each individual, we calculate two entities firstly: one is $n_{i}$, the number of individuals which dominate the individual $i$, and the other is $\boldsymbol{S}_{i}$, a set of individuals that the individual $i$ dominates. The sorting steps are as follows.

Step 1: Find out the individuals with $n_{i}=0$ in the initial population, put them into the set $\boldsymbol{F}_{1}$ as the first non-dominated front, and set the non-dominated rank of all the individuals in this front as $i_{\text {rank }}=1$.

Step 2: For each individual $i$ in $\boldsymbol{F}_{1}$, visit every individual $l$ in $\boldsymbol{S}_{i}$, and execute $n_{l}=n_{l}-1$. For any individual $l$, when $n_{l}$ becomes zero, put it into the set $\boldsymbol{F}_{2}$ as the second nondomination front. Set the non-dominated rank of all the individuals in this front as $i_{\text {rank }}=2$.

Step 3: For the set $\boldsymbol{F}_{2}$, repeat step 2. Put the individuals with $n_{l}=0$ into the set $\boldsymbol{F}_{3}$ as the third non-dominated front. Set the non-dominated rank of all the individuals in this front as $i_{\text {rank }}=3$. Continue this process until all fronts are identified.

\subsubsection{Individual crowding distance design}

The design of individual crowding distance is proposed in NSGA-II to sort the individuals with the same $i_{\text {rank }}$. The crowding distance of the individual $i$ is defined as the distance between its two adjacent individuals $i+1$ and $i-1$ in the target space. The calculating steps are as follows.

Step 1: Initialize the crowding distance of individuals in the same front, set $L[i]=0$.

Step 2: Sort the individuals in the same front in ascending order of the value of the $m^{\text {th }}$ objective function.

Step 3: The boundary individuals are assigned an infinite value $W$ :

$\left\{\begin{array}{c}L\left[i_{\min }\right]=L\left[i_{\min }\right]+W \\ L\left[i_{\max }\right]=L\left[i_{\max }\right]+W\end{array}\right.$

where $i_{\max }$ and $i_{\min }$ are the individuals with the maximum and minimum value of the $m^{\text {th }}$ objective function, respectively. 
Step 4: For all the intermediate individuals, the crowding distance can be derived from (21).

$L[i]=L[i]+\frac{f_{m}^{i+1}-f_{m}^{i-1}}{f_{m}^{\max }-f_{m}^{\min }}$

where $f_{m}^{i+1}$ and $f_{m}^{i-1}$ are the values of the $m^{\text {th }}$ objective function corresponding to the individual $i+1$ and $i-1$; $f_{m}^{\max }$ and $f_{m}^{\min }$ are the maximum and minimum value of the $m^{\text {th }}$ objective function, respectively.

Step 5: For other objective functions, repeat step 2-4. Then $L[i]$, the crowding distance of the individual $i$, can be obtained.

Prefer the individuals with the larger crowding distance, and then the solutions can be uniformly distributed in the target space. Thus, the diversity of the individuals can be preserved.

\subsubsection{Elitism strategy}

Elitism strategy can put the superior individuals of the parent generation into the child generation, which avoids the missing of the Pareto optimal solutions. The processing steps are as follows.

Step 1: Combine the parent generation $\boldsymbol{P}_{t}$ and the child generation $\boldsymbol{Q}_{t}$ to form one population $\boldsymbol{R}_{t}=\boldsymbol{P}_{t} \cup \boldsymbol{Q}_{t}$, then apply the fast non-dominated sorting approach on $\boldsymbol{R}_{t}$, and then calculate the individual crowding distance.

Step 2: Put the individuals of $\boldsymbol{R}_{t}$ into the new parent generation $\boldsymbol{P}_{t+1}$ in ascending order of the non-dominated rank. Stop it until the number of individual of $\boldsymbol{P}_{t+1}$ exceeds $N$ (the number of the population), when all the individuals of $\boldsymbol{F}_{j}$ are added.

Step 3: Put the individuals of $\boldsymbol{F}_{j}$ into $\boldsymbol{P}_{t+1}$ in descending order of the individual crowding distance, until the number of $\boldsymbol{P}_{t+1}$ equals $N$.

\subsubsection{Tournament selection}

The selection approach should guide the optimization process towards the Pareto optimal solutions and keep the distribution and diversity of the individuals. The results of the tournament selection are the chosen individuals used to generate the child generation.

Tournament selection compares the individuals of parent generation in a random pairing mode. The individual $i$ is thought to be superior to the individual $j$ if $i_{\text {rank }}<j_{\text {rank }}$ or $i_{\text {rank }}=j_{\text {rank }}$ and $L[i]>L[j]$. That's to say, for two individuals with different non-dominated ranks, we prefer the individual with the lower rank; if both individuals belong to the same front, we prefer the individual that is located in the less crowded region.

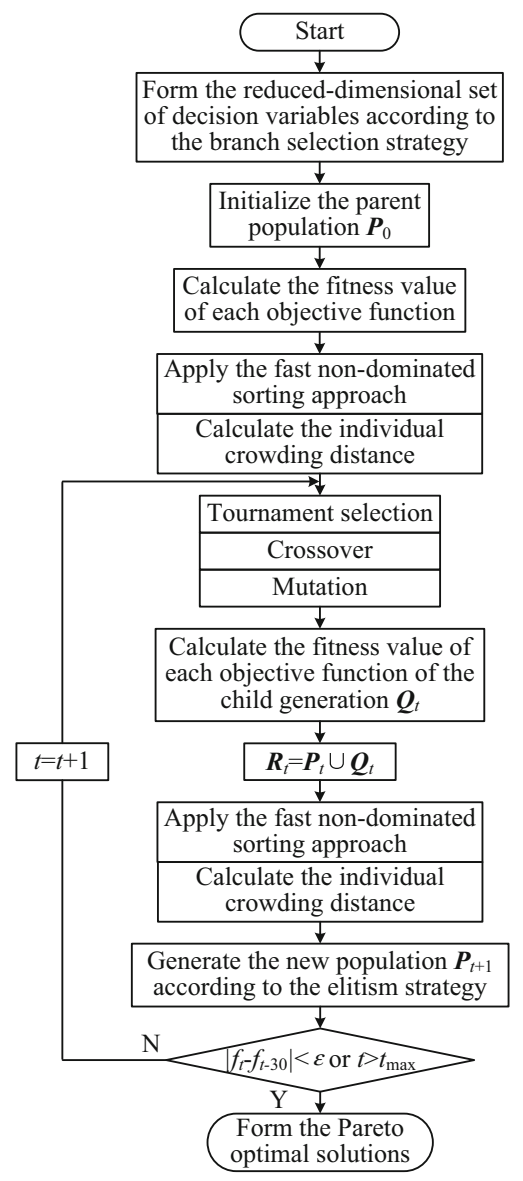

Fig. 4 Flow chart of coordinated optimization method

\subsubsection{Crossover and mutation}

Cooperation of crossover and mutation can give genetic algorithm good local and global search performances. Single point crossover and random mutation are performed on the population obtained by tournament selection, then the child population $\boldsymbol{Q}_{t}$ can be generated.

\subsection{The process of method}

In summary, the process of coordinated optimization method is shown in Fig. 4. Where $f_{t}$ is the average of the fitness values of all the individuals in the first front; $t_{\max }$ is the maximum number of evolution generations.

\section{Simulation of planning power system}

Taking a planning power system for example, the feasibility and speediness of this method is validated. Considering a 5\% margin, for the breakers with maximum interruptive current of $63 \mathrm{kA}$, the short circuit current upper limit is set to $59.85 \mathrm{kA}$. The impedance value of current limiting reactor is 


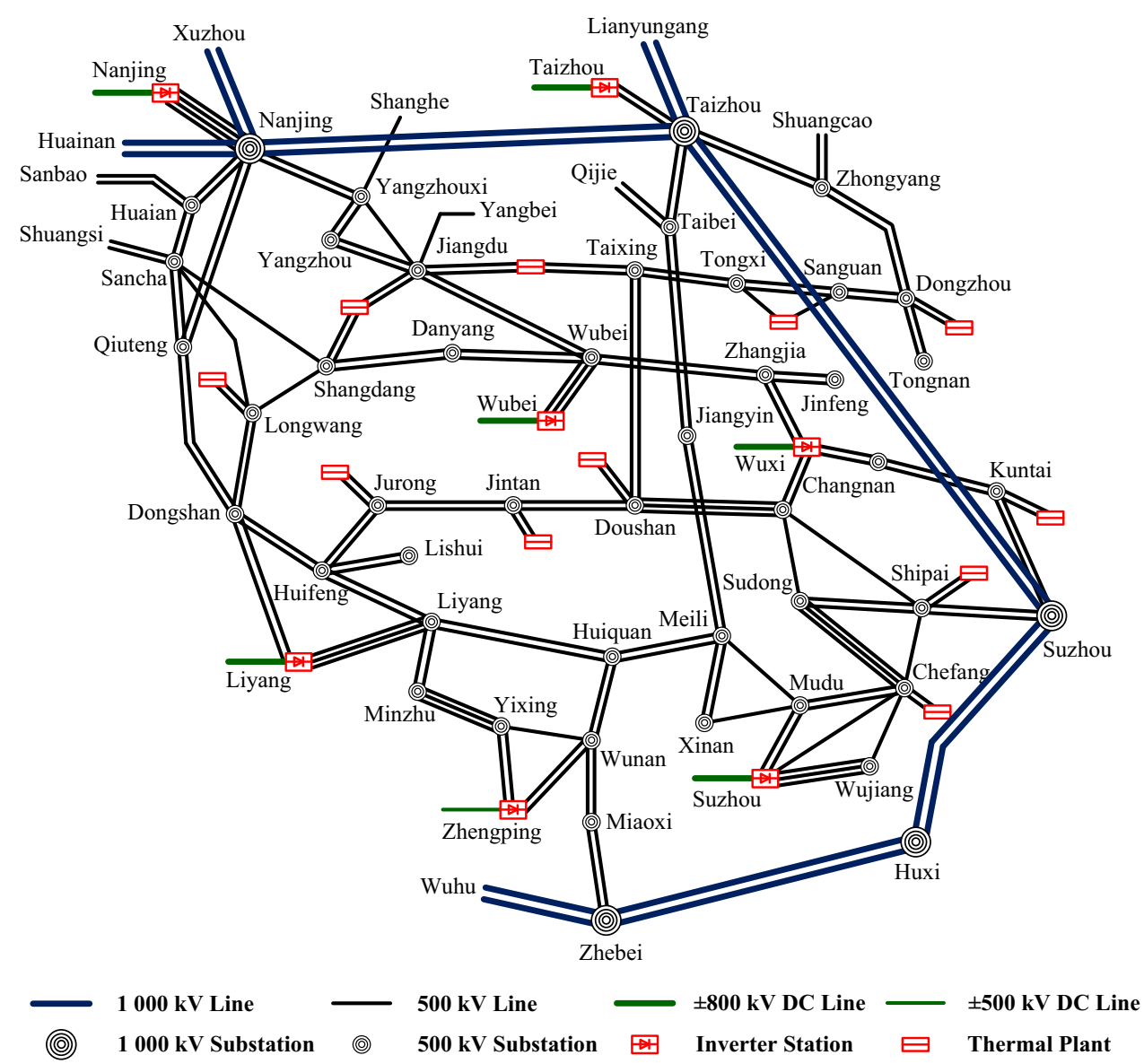

Fig. 5 Regional power grid structure in China

Table 1 MISCR of the multi-infeed DC system

\begin{tabular}{llll}
\hline Inverter Bus & Voltage/kV & Capacity/MW & MISCR \\
\hline Suzhou & \pm 800 & Bipolar 7200 & 2.5618 \\
Zhengping & \pm 500 & Bipolar 3000 & 2.9589 \\
Liyang & \pm 800 & Bipolar 8000 & 2.4035 \\
Nanjing & \pm 800 & Bipolar 8000 & 2.7150 \\
Wubei & \pm 800 & Bipolar 8000 & 2.5708 \\
Taizhou & \pm 800 & Bipolar 8000 & 3.2051 \\
Wuxi & \pm 800 & Bipolar 8000 & 2.9194 \\
\hline
\end{tabular}

set to the range of $0-10 \Omega$. The minimum multi-infeed shortcircuit ratio is set to 2.0 [18]. The cost factors of opening the line are as follows: $k_{a s}=60, k_{b s}=0$, and the cost factors of installing the current limiting reactor are as follows: $k_{a s}=625, k_{b s}=25[13]$.

The structure of regional power grid in China is shown in Fig. 5. There are 7 DC inverter stations (Suzhou, Zhengping, Liyang, Nanjing, Wubei, Taizhou and Wuxi) in this regional power grid, forming a typical multi-infeed DC system. The multi-infeed short-circuit ratio of this system are shown in Table 1.
Under this operating mode, the three-phase short circuit currents of $500 \mathrm{kV}$ buses in Shipai, Changnan, Suzhou, Sudong, Doushan and Chefang substation are 77.43, 73.82, $70.64,68.59,67.28$ and $66.46 \mathrm{kA}$, respectively. All of them are exceeding the short circuit current upper limit (59.85 $\mathrm{kA})$. According to the branch selection strategy, all the lines in the network are arranged in the descending order of the integrated sensitivity. The results are shown in Table 2 . The lines with $\mu_{l}>0.01$ are put into the reduced-dimensional set, and the lines with $\mu_{l}<0.01$ have no competitiveness.

The population size is set to 100 , the maximum generation is set to 500 , and the crossover rate is set to 0.9. In order to prevent the deviation of single optimization caused by random factors, 400 times calculations are carried out under different calculating parameters. The statistical results are shown in Table 3. The length of full-dimensional individual is 90 , the length of reduced-dimensional individual is 44 , and $P / \%$ is the average probability that the solutions of each calculation in accordance with the Pareto optimal solutions of 400 times calculations. As is shown in Table 3, the optimization using the reduced-dimensional 
Table 2 Integrated sensitivity ranking of current limiting measures

\begin{tabular}{|c|c|c|c|c|c|}
\hline No. & Line name & $\mu_{l}$ & No. & Line name & $\mu_{l}$ \\
\hline 1 & Shipai-Changnan & 1.000 & 23 & Kuntai-Suzhou II & 0.076 \\
\hline 2 & Shipai-Suzhou I & 0.458 & 24 & Wubei-Zhangjia I & 0.073 \\
\hline 3 & Shipai-Suzhou II & 0.458 & 25 & Wubei-Zhangjia II & 0.073 \\
\hline 4 & Changnan-Sudong & 0.381 & 26 & Meili-Huiquan I & 0.046 \\
\hline 5 & Shipai-Sudong I & 0.321 & 27 & Meili-Huiquan II & 0.046 \\
\hline 6 & Shipai-Sudong II & 0.321 & 28 & Changnan-Kuntai I & 0.044 \\
\hline 7 & Doushan-Changnan I & 0.219 & 29 & Changnan-Kuntai II & 0.044 \\
\hline 8 & Doushan-Changnan II & 0.219 & 30 & Meili-Xinan I & 0.041 \\
\hline 9 & Doushan-Changnan III & 0.219 & 31 & Meili-Xinan II & 0.041 \\
\hline 10 & Xinan-Mudu & 0.208 & 32 & Tongxi-Taixing I & 0.032 \\
\hline 11 & Shiapai-Chefang & 0.197 & 33 & Tongxi-Taixing II & 0.032 \\
\hline 12 & Taixing-Doushan I & 0.195 & 34 & Jurong-Jintan I & 0.030 \\
\hline 13 & Taixing-Doushan II & 0.195 & 35 & Jurong-Jintan II & 0.030 \\
\hline 14 & Chefang-Sudong I & 0.142 & 36 & Taibei-Jiangyin I & 0.027 \\
\hline 15 & Chefang-Sudong II & 0.142 & 37 & Taibei-Jiangyin II & 0.027 \\
\hline 16 & Chefang-Sudong III & 0.142 & 38 & Meili-Jiangyin I & 0.019 \\
\hline 17 & Meili-Mudu & 0.130 & 39 & Meili-Jiangyin II & 0.019 \\
\hline 18 & Jintan-Doushan I & 0.101 & 40 & Chefang-Wujiang & 0.014 \\
\hline 19 & Jintan-Doushan II & 0.101 & 41 & Huifeng-Jurong I & 0.012 \\
\hline 20 & Chefang-Mudu I & 0.094 & 42 & Huifeng-Jurong II & 0.012 \\
\hline 21 & Chefang-Mudu II & 0.094 & 43 & Sanguan-Tongxi I & 0.011 \\
\hline 22 & Kuntai-Suzhou I & 0.076 & 44 & Sanguan-Tongxi II & 0.011 \\
\hline
\end{tabular}

Table 3 Statistical results under different calculating parameters

\begin{tabular}{llllll}
\hline No. & $\begin{array}{l}\text { Individual } \\
\text { dimension }\end{array}$ & $\begin{array}{l}\text { Mutation } \\
\text { rate }\end{array}$ & $\begin{array}{l}\text { Calculating } \\
\text { times }\end{array}$ & $\begin{array}{l}\text { Average } \\
\text { converged } \\
\text { generation }\end{array}$ & $P / \%$ \\
\hline 1 & $\begin{array}{c}\text { Reduced- } \\
\text { dimensional }\end{array}$ & 0.4 & 100 & 69 & 85 \\
2 & $\begin{array}{c}\text { Full-dimensional } \\
3\end{array}$ & 0.4 & 100 & 398 & 46 \\
& $\begin{array}{c}\text { Reduced- } \\
\text { dimensional }\end{array}$ & 0.2 & 100 & 60 & 74 \\
4 & Full-dimensional & 0.2 & 100 & 372 & 33 \\
\hline
\end{tabular}

decision variables has less converged generation and better converged solutions. And using a larger mutation rate could get a closer solution compared with the Pareto optimal solutions, although it increases the converged generation.

The method combining the reduced-dimensional decision variables and a larger mutation rate is adopted to solve this optimization model. An optimization calculation is converged at the 70th generation. In order to keep the distribution and diversity of the individuals, sort the solutions in descending order of the crowding distance. The first 5 Pareto optimal solutions are shown in Table 4, and the corresponding fitness values are shown in Table 5. It
Table 4 Pareto optimal solutions

\begin{tabular}{|c|c|c|}
\hline \multirow[t]{2}{*}{ Scheme } & \multicolumn{2}{|c|}{ Current limiting measure } \\
\hline & Open the line & Install the current limiting reactor \\
\hline 1 & $\begin{array}{l}\text { Shipai-Changnan } \\
\text { Changnan-Sudong } \\
\text { Xinan-Mudu } \\
\text { Meili-Mudu }\end{array}$ & None \\
\hline 2 & $\begin{array}{l}\text { Shipai-Changnan } \\
\text { Doushan-Changnan I }\end{array}$ & $\begin{array}{l}\text { Shipai-Suzhou I Line }+9 \Omega \\
\text { Shipai-Suzhou II Line }+10 \Omega\end{array}$ \\
\hline 3 & $\begin{array}{l}\text { Shipai-Changnan } \\
\text { Xinan-Mudu } \\
\text { Meili-Mudu } \\
\text { Shipai-Suzhou II }\end{array}$ & None \\
\hline 4 & $\begin{array}{l}\text { Shipai-Changnan } \\
\text { Doushan-Changnan I } \\
\text { Shipai-Suzhou I }\end{array}$ & Shipai-Suzhou II Line $+2 \Omega$ \\
\hline 5 & $\begin{array}{l}\text { Shipai-Changnan } \\
\text { Changnan-Sudong } \\
\text { Shipai-Suzhou I }\end{array}$ & Shipai-Suzhou II Line $+2 \Omega$ \\
\hline
\end{tabular}

can be concluded that the total cost $f_{1}$ and the short circuit capacity margin $f_{2}$ are contradictory to each other. Taking scheme 1 and 2 for examples, the total cost of scheme 1 is 
Table 5 Fitness values of Pareto optimal solutions

\begin{tabular}{lcll}
\hline Scheme & \multicolumn{2}{l}{ Fitness value } \\
\cline { 2 - 4 } & $f_{1}$ & $f_{2}$ & $f_{3}$ \\
\hline 1 & 240 & 17.232 & -24.615 \\
2 & 1845 & 10.643 & -24.264 \\
3 & 240 & 15.444 & -24.582 \\
4 & 855 & 11.674 & -24.244 \\
5 & 855 & 12.529 & -24.308 \\
\hline
\end{tabular}

the lowest and its short circuit capacity margin is largest, however, scheme 2 has the largest total cost and the smallest short circuit capacity margin. The contradiction between $f_{1}$ and $f_{2}$ is determined by the characteristics of current limiting measures. Opening the line is the one with best effect and lowest cost, but it will significantly reduce the tightness of network. Installing the current limiting reactor has a smoother current limiting effect and can keep the tightness of network, but the cost is high.

The weighted multi-infeed short-circuit ratio $\left(-f_{3}\right)$ of scheme 1 and 3 are larger than the one (24.352) before the optimization is carried out. It is proved by the numerical simulations that such two schemes give the receiving-end AC system a stronger voltage support capacity to multiple DCs. When the same faults occur, the recovery rate of system voltage and DC power of scheme 1 is the fastest between all the schemes. The multi-infeed short-circuit ratio and short circuit current of all the schemes are shown in Table 6 and Table 7. The power flow and stability calculation demonstrates that all the schemes can satisfy the " $N-1$ " constraints.

If the single-objective optimization for the cost of current limiting measures is carried out, only scheme 1 and 3 in Table 4 can be obtained. The presented method in this paper can provide the Pareto optimal solutions. Therefore, the decision-makers can choose the one they prefer. For example, if the requirement is to invest the minimum cost of current limiting measures, scheme 1 and 3 can be chosen; if the requirement is to maintain the integrity of the electrical power grid, scheme 2 can be chosen; if the stronger voltage support capacity of AC system to multiple DCs is required, scheme 1 and 3 are the better choices; if the optimizing balance of all the objective functions is required, scheme 4 and 5 may be the preferred ones.

\section{Conclusion}

In order to solve the problems of exceeding short circuit current and improve the voltage stability in multi-infeed DC system, a coordinated optimization method is presented in this paper. The simulation results of the planning regional power grid in China demonstrated the feasibility and efficiency of the method. The branch selection strategy considering sensitivity ranking can effectively reduce the search range of decision variables, therefore avoid the optimization falling into the curse of dimensionality.

Table 6 MISCR of different schemes

\begin{tabular}{llllll}
\hline Inverter bus & Scheme 1 & Scheme 2 & Scheme 3 & Scheme 4 & Scheme 5 \\
\hline Suzhou & 2.3340 & 2.5505 & 2.3319 & 2.5497 & 2.9614 \\
Zhengping & 3.1283 & 2.9616 & 3.1271 & 2.4097 & 2.9637 \\
Liyang & 2.4651 & 2.4098 & 2.4627 & 2.7113 & 2.4078 \\
Nanjing & 2.7282 & 2.7113 & 2.7277 & 3.5459 & 2.7104 \\
Wubei & 2.5490 & 2.5459 & 3.5438 & 2.549 & 3.2047 \\
Taizhou & 3.2919 & 3.2046 & 2.82029 & 2.8523 & 2.8819 \\
Wuxi & 2.8376 & 2.8521 & &
\end{tabular}

Table 7 Short circuit currents of different schemes

\begin{tabular}{|c|c|c|c|c|c|}
\hline \multirow[t]{2}{*}{ Bus name } & \multicolumn{5}{|c|}{ Actual short circuit current (kA) } \\
\hline & Scheme 1 & Scheme 2 & Scheme 3 & Scheme 4 & Scheme 5 \\
\hline Shipai & 49.13 & 58.79 & 52.47 & 58.47 & 49.93 \\
\hline Changnan & 48.77 & 59.37 & 59.74 & 59.37 & 48.62 \\
\hline Suzhou & 58.88 & 59.64 & 58.96 & 59.32 & 58.85 \\
\hline Sudong & 41.46 & 59.75 & 50.42 & 59.74 & 46.82 \\
\hline Doushan & 51.77 & 57.66 & 59.28 & 57.65 & 51.64 \\
\hline Chefang & 39.99 & 59.11 & 46.53 & 58.93 & 48.84 \\
\hline
\end{tabular}


Combining this strategy with NSGA-II can improve the convergence characteristics of the optimizing process. Considering the total cost, short circuit capacity margin and weighted multi-infeed short-circuit ratio, the Pareto optimal schemes can be obtained, which provides the decision-makers with more comprehensive and enriched choices.

Acknowledgment This work was supported by State Grid Corporation of China, Major Projects on Planning and Operation Control of Large Scale Grid under Grant SGCC-MPLG020-2012.

Open Access This article is distributed under the terms of the Creative Commons Attribution License which permits any use, distribution, and reproduction in any medium, provided the original author(s) and the source are credited.

\section{References}

[1] Zhang W, Liu YT (2008) Multi-objective reactive power and voltage control based on fuzzy optimization strategy and fuzzy adaptive particle swarm. Int J Electr Power Energ Syst 30(9): 525-532

[2] Granelli G, Montagna M, Zanellini F et al (2006) A genetic algorithm-based procedure to optimize system topology against parallel flows. IEEE Trans Power Syst 21(1):333-340

[3] Zhao CY, Guan YP (2013) Unified stochastic and robust unit commitment. IEEE Trans Power Syst 28(3):3353-3361

[4] Liu YT, Zhang P, Qiu XZ (2002) Optimal volt/var control in distribution systems. Int J Electr power Energ Syst 24(4): 271-276

[5] Hongesombut K, Mitani Y, Tsuji K (2003) Optimal location assignment and design of superconducting fault current limiters applied to loop power systems. IEEE Trans Appl Supercon 13(2-2):1828-1831

[6] Yannibelli V, Amandi A (2013) Hybridizing a multi-objective simulated annealing algorithm with a multi-objective evolutionary algorithm to solve a multi-objective project scheduling problem. Expert Syst Appl 40(7):2421-2434

[7] Deb K, Pratap A, Agarwal S et al (2002) A fast and elitist multiobjective genetic algorithm: NSGA-II. IEEE Trans Evolut Comput 6(2):182-197

[8] Shahriari SAA, Yazdian A, Haghifam MR (2009) Fault current limiter allocation and sizing in distribution system in presence of distributed generation. In: Proceedings of the 2009 IEEE power and energy society general meeting (PES'09), Calgary, 26-30 Jul 2009, 6 pp

[9] Kim SY, Bae IS, Kim JO (2010) An optimal location for superconducting fault current limiter considering distribution reliability. In: Proceedings of the 2010 IEEE power and energy society general meeting (PES'10), Minneapolis, 25-29 Jul 2010, $5 \mathrm{pp}$

[10] Nagata M, Tanaka K, Taniguchi H (2001) FCL location selection in large scale power system. IEEE Trans Appl Supercon 11(1-2):2489-2494

[11] Teng JH, Lu CN (2010) Optimum fault current limiter placement with search space reduction technique. IET Gener Transm Distrib 4(4):485-494
[12] Namchoat S, Hoonchareon N (2013) Optimal bus splitting for short-circuit current limitation in metropolitan area. In: Proceedings of the 10th international conference on electrical engineering/electronics, computer, telecommunications and information technology (ECTI-CON'13), Krabi, 15-17 May 2013, 5 pp

[13] Chen LL, Huang MX, Wu JY, et al (2010) An optimal strategy for short circuit current limiter deployment. In: Proceedings of the 2010 Asia-Pacific power and energy engineering conference (APPEEC'10), Chengdu, 28-31 Mar 2010, 4 pp

[14] Aik DLH, Andersson G (1997) Voltage stability analysis of multi-infeed HVDC systems. IEEE Trans Power Deliver 12(3):1309-1318

[15] Bui LX, Sood VK, Laurin S (1991) Dynamic interactions between HVDC systems connected to AC buses in close proximity. IEEE Trans Power Deliver 6(1):223-230

[16] Aik DLH, Andersson G (2013) Analysis of voltage and power interactions in multi-infeed HVDC systems. IEEE Trans Power Deliver 28(2):816-824

[17] IEEE Std 1204-1997. IEEE guide for planning DC links terminating at AC locations having low short-circuit capacities. 1997

[18] CIGRE Working Group B4.41 (2008) Systems with multiple DC infeed. CIGRE, Paris

[19] Lin WF, Tong Y, Bu GQ, et al (2010) Voltage stability analysis of multi-infeed AC/DC power system based on multi-infeed short circuit ratio. In: Proceedings of the 2010 international conference on power system technology (POWERCON'10), Hangzhou, 24-28 Oct 2010, 6 pp

[20] de Toledo PF, Bergdahl B, Asplund G (2005) Multiple infeed short circuit ratio-aspects related to multiple HVDC into one AC network. In: Proceedings of the 2005 IEEE/PES transmission and distribution conference and exhibition: Asia and Pacific, Dalian, 14-18 Aug 2005, 6 pp

[21] Tada Y, Okamoto H, Kurita A et al (1998) Analytical methods for determining a system configuration acceptable from viewpoints of both short circuit current and voltage stability. Electr Eng JPN 124(3):30-39

[22] Huang HY, Xu Z, Lin X (2012) Improving performance of multi-infeed HVDC systems using grid dynamic segmentation technique based on fault current limiters. IEEE Trans Power Syst 27(3):1664-1672

Dong YANG Received the B.E. and Ph.D. degrees from Shandong University in 2007 and 2013, respectively. He is now an engineer in State Grid Shandong Electric Power Research Institute. His research interests include power system operation and planning.

Kang ZHAO Received the B.E. degree from Huazhong University of Science and Technology in 2013. He is now a M.E. candidate in Shandong University. His research interests include power system operation and planning.

Yutian LIU received the B.E. and M.E. degrees from Shandong University in 1984 and 1990, respectively, and the Ph.D. degree from Xi'an Jiaotong University in 1994. He is now a professor with the School of Electrical Engineering, Shandong University. His research interests include renewable energy sources integrated into smart grid, power system security and stability, and power system restoration. 